\title{
Crossing the Line: Crowd Counting by Integer Programming with Local Features
}

\author{
Zheng Ma \\ Antoni B. Chan \\ Department of Computer Science \\ City University of Hong Kong \\ zhengma2-c@my.cityu.edu.hk, abchan@cityu.edu.hk
}

\begin{abstract}
We propose an integer programming method for estimating the instantaneous count of pedestrians crossing a line of interest in a video sequence. Through a line sampling process, the video is first converted into a temporal slice image. Next, the number of people is estimated in a set of overlapping sliding windows on the temporal slice image, using a regression function that maps from local features to a count. Given that count in a sliding window is the sum of the instantaneous counts in the corresponding time interval, an integer programming method is proposed to recover the number of pedestrians crossing the line of interest in each frame. Integrating over a specific time interval yields the cumulative count of pedestrian crossing the line. Compared with current methods for line counting, our proposed approach achieves state-of-the-art performance on several challenging crowd video datasets.
\end{abstract}

\section{Introduction}

The goal of crowd counting is to estimate the number of people in a region of interest (ROI counting), or passing through a line of interest (LOI counting) in video. Crowd counting has many potential real-world applications, including surveillance (e.g., detecting abnormally large crowds, and controlling the number of people in a region), resource management (counting the number of people entering and exiting), and urban planning (identifying the flow rate of people around an area). Beyond people, these counting methods can also be applied to other objects, such as animals passing through a particular boundary, blood cells flowing through a blood vessel under a microscope, and the rate of car traffic. Therefore crowd counting is a crucial topic in video surveillance and other related fields. However, it is still a challenging task because of several factors: 1) in crowded scenes, occlusion between pedestrians is common, especially for large groups in confined areas; 2) the perspective of the scene causes people to appear larger and move faster when they are close to the camera. These problems are especially prominent in "high-angle" camera (a)

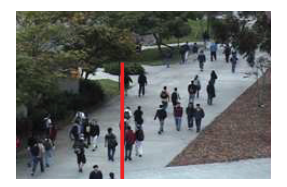

(b)

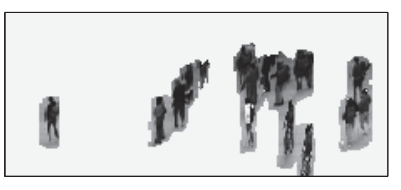

(c)

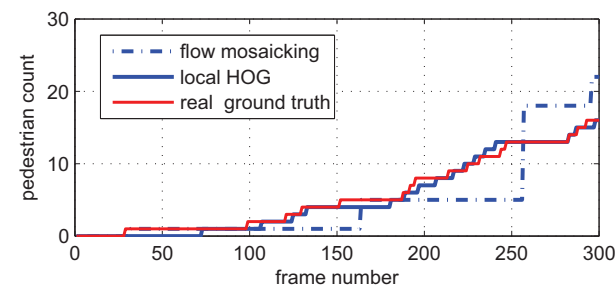

Figure 1. Line counting example: a) crowd scene and line-ofinterest; b) temporal slice of the scene; c) Flow-mosaicking [1] result where a large blob leads to a big jump in the cumulative count. In contrast, our method can predict instantaneous counts better, yielding a better cumulative prediction over time.

views (where the camera looks down at an angle), which are typical of outdoor surveillance cameras.

Most previous approaches [2-6] focus on solving the ROI counting problem, and are based on the countingby-regression framework, where features extracted from the ROI are directly regressed to the number of people. By bypassing intermediate steps, such as people detection, which can be error-prone on large crowds with severe occlusion, these counting-by-regression methods achieve accurate counts even on sizable crowds. In this paper, we focus on LOI counting, which has received relatively less attention so far. The goal of LOI counting is to count the number of people crossing a line (or visual gate) in the video (see Fig. 1a for example). In particular, the aim is to estimate both the cumulative count, i.e., the total count since the start of the video, and the instantaneous count, i.e., the count at any particular time or short temporal window. A naive approach to LOI counting is to apply ROI counting on the regions on each side of the LOI, and take the count difference. However, this LOI count will be erroneous when people enter and exit the ROIs at the same time, since the number of people in the regions remains the same.

Current state-of-the-art LOI counting approaches (e.g. [1]) are based on extracting and counting crowd blobs from 
(a)
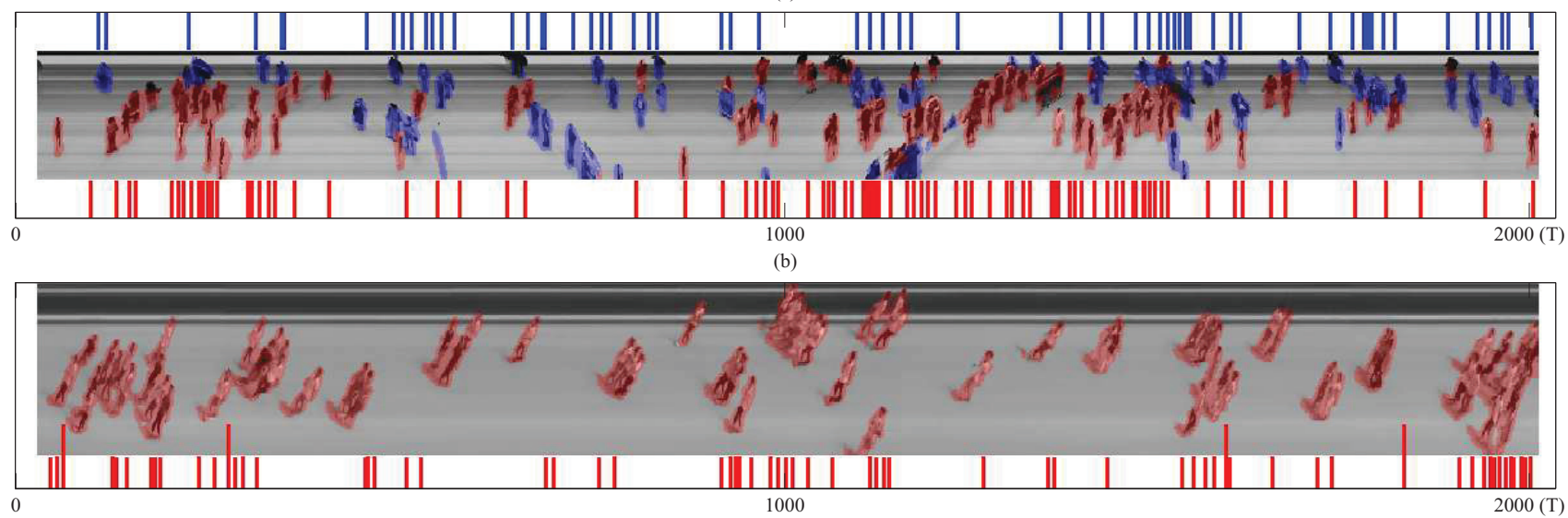

Figure 2. Results of instantaneous count estimation on: a) UCSD and b) LHI datasets. The image is a temporal-slice of the video on the LOI. The red and blue segments correspond to crowds moving in different directions, and the instantaneous count estimates appear above and below the image.

a temporal slice of the video (e.g., the y-t slice of the video volume). However, there are several drawbacks of these "blob-centric" methods: 1) because the blob is not counted until it has completely crossed the line, large blobs (e.g., containing more than 10 people) yield big jumps in the cumulative count, which leads to poor instantaneous count estimates (see Fig. 1c); 2) the counts in these large blobs are not accurate, due to severe occlusion [1]; 3) current evaluation methods for blob-based methods are based on the ground-truth people in the blob, not the actual people passing the line - hence, it is difficult to assess errors due to segmentation failure of the blob. Moreover, these methods typically require spatial-temporal normalization to handle the differences in pedestrian size due to the camera perspective and pedestrian velocity. Current perspective normalization methods $[2,7]$ require marking a reference person in different positions in the video. For arbitrary videos (e.g., from the internet), these normalization techniques cannot be applied if no suitable reference exists.

To address the above problems, we propose a novel line counting algorithm that estimates instantaneous people counts using local-level features and regression without perspective normalization (see Fig. 2 for examples). The contributions of this paper are three-fold. First, to overcome the drawbacks of "blob-centric" methods, we propose an integer programming approach to estimate the instantaneous counts on the LOI, from a set of ROI counts in the temporal slice image. The cumulative counts of our method are smoother and more accurate than "blob-centric" methods. Second, we introduce a novel local histogram-of-orientedgradients (HOG) feature, which is robust to the effects of perspective and velocity and yields accurate counts even without spatial-temporal normalization. Third, we demonstrate experimentally that our method can achieve state-ofthe-art results for both cumulative and instantaneous LOI counts on two challenging datasets.

\section{Related work}

Counting-by-regression methods focus on either counting people in a region-of-interest (ROI), or counting people passing through a line-of-interest (LOI). For ROI counting, features are extracted from each crowd segment in an image, and a regression function maps between the feature space and the number of people in the segment. Typically low-level global features are extracted from the crowd segment, internal edges, and textures $[1,2,4,6]$. The segment area is a prototypical feature that can indicate the total number of pedestrians in the segment. [2] shows that there is a near linear relationship between the segment area and the number of pedestrian, as long as the feature extraction process properly weights each pixel according to the perspective of the scene. [2] also provides a method to generate a perspective map, by manually labeling a reference person at opposite positions in the scene. Low-level features can also be extracted from each crowd blob, i.e., an individual connected-component in the segment, which contains several pedestrians [5, 6]. Regression methods include Gaussian process regression [8] or Bayesian Poisson regression [4], which are both kernel methods that can estimate non-linear functions. [9] proposes an alternative approach to ROI counting, using pixel-wise density learning. The crowd density at each pixel is regressed from the feature vector, and the number of pedestrians in a ROI is obtained by integrating over the crowd density map.

Line-of-interest (LOI) counting essentially estimates the number of people in a temporal-slice image (e.g., the y-t slice of the video volume), the result of which represents the number of people passing through the line within that time window. However, with the basic temporal slice, people moving at fast speeds will have fewer pixels than 
those moving slowly, thus confounding the regression function. The flow-mosaiking framework [1] corrects for this by changing the thickness of the line, based on the the average velocity of the pixels in the crowd blob, resulting in a "flow mosaic". [7] is used for perspective normalization, and the count in each blob is estimated from low-level features. The blob count can only be estimated after the blob has passed the line, and hence large jumps in the cumulative count can occur, and instantaneous counts (indicating when each person passes the line) are not possible. In contrast to [1], our proposed approach performs ROI counting on windows in the temporal slice image, and uses integer programming to recover the instantaneous count on the line.

For both ROI and LOI counting, global- and bloblevel features are very sensitive to the perspective (spatiotemporal) normalization, especially in scenes with large depth changes. In our experiments, the accuracy of counting using low-level features drops significantly without normalization, whereas the accuracy using our local HOG feature remains at a similar level. [9] also uses local features, based on random forest [9] or SIFT features [10], but still requires perspective normalization.

Finally, counting can also be performed using people detection methods [11-13], which are based on "individualcentric" features, i.e., features describing the whole person, such as the HOG descriptor of a whole person [11]. The deformable part-based model (DPM) [13] also builds a HOG descriptor of a whole person, by using a more flexible layout model for the spatial relationship between HOG parts at different scales. While this results in a model that is better adapted to varying poses of a single person, it still has problems in detecting partially-occluded people in groups. In contrast, by removing the layout model, our local HOG representation is better able to handle occlusions.

\section{Local HOG feature}

Fig. 3a shows an example of a temporal-slice image with a sizable crowd walking in two directions. Due to the camera tilt angle, which is nearly 45 degree, the occlusion of pedestrians is heavy. The torsos or the legs of the occluded pedestrians are not visible in most cases. Hence, the standard histogram-of-oriented-gradients (HOG) feature and detector [11] will not work well in this scenario. In this paper, we propose a local HOG descriptor for crowd counting. In contrast to the standard HOG, which is a descriptor of a whole person, the local HOG can describe parts of the person independently. As a result, in crowded scenes, meaningful descriptors can still be extracted from partiallyoccluded people.

\subsection{Local HOG descriptor}

We define a local HOG descriptor as one "block" of the standard HOG feature, extracted from a $8 \times 8$ image

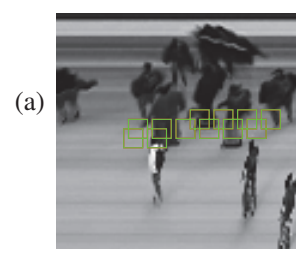

(b)
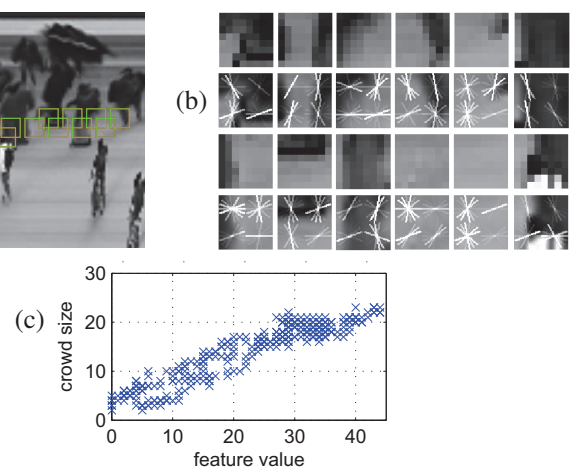

Figure 3. Examples of local HOG features: a) temporal-slice image; b) image patches and their local HOG features; c) one bin of the bag-of-words histogram versus crowd size.

patch. Each block contains four $4 \times 4$ "spatial cells", from which the local histogram of oriented gradients is extracted. Fig. $3 \mathrm{~b}$ presents examples of the local HOG features extracted from a crowd in the temporal slice image. Note how the local HOG feature can represent the head-shoulders, side, or legs and feet of a person. We also considered rectangular image patches (e.g., $8 \times 16)$ and other sizes, and found that the $8 \times 8$ image patches yield the best performance in the experiments. Finally, we considered applying a weight to the gradient magnitudes using a spatial Gaussian kernel (similar to the SIFT descriptor [10]), but this did not increase the counting accuracy.

\subsection{Global descriptor of local HOG features}

The number of extracted local HOG features depends on the size of the crowd segments in each video frame, with potentially hundreds of local features extracted per frame due to dense sampling. Hence, further processing is required to summarize the set of local features into a concise feature vector, which describes the crowd. The set of local HOG features is summarized with a bag-of-words histogram, where each bin represents the number of times a local HOG codeword appears in the image. Fig. $3 \mathrm{c}$ plots the value of one bin of the histogram versus the number of people in the crowd segment. The bin value varies linearly with the number of people, which suggests that the bagof-words of local HOG can be a suitable feature for crowd counting. Finally, we do not apply histogram normalization methods (e.g., TF, TF-IDF). Normalization will obfuscate the absolute number of codewords in the segment, making histograms from large crowds similar to those from small crowds, which confounds the regression function.

\section{Line counting framework}

In this section, we propose our line counting framework, which is illustrated in Fig. 4. Given an input video sequence, the video is first segmented into crowds of interest, e.g., corresponding to people moving in different directions. 


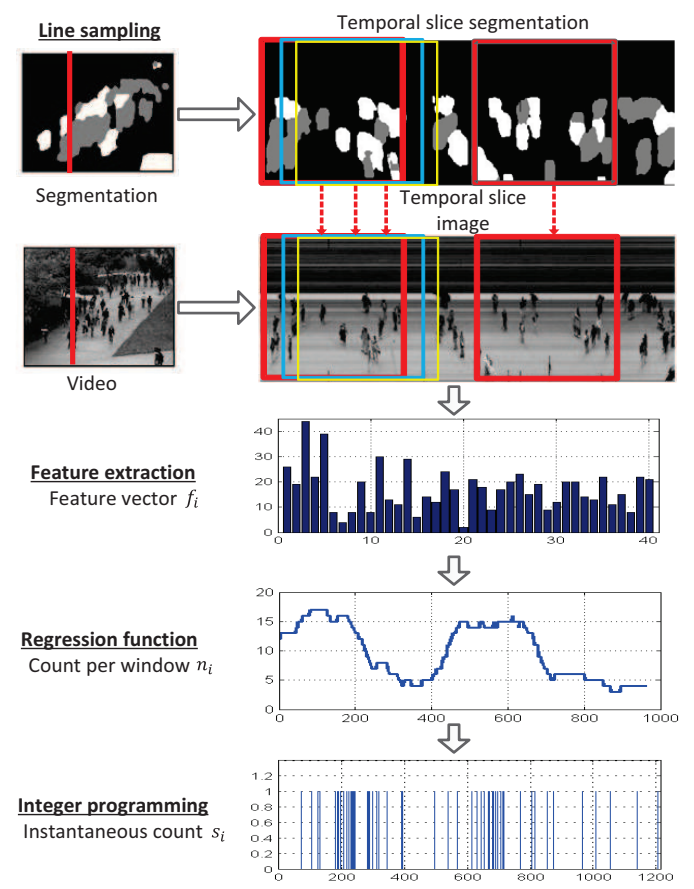

Figure 4. The proposed line counting framework.

A temporal slice image and temporal slice segmentation are formed by sampling the LOI over time. Next, a sliding window is placed over the temporal slice, forming a set of temporal ROIs. Features are extracted from each temporal ROI, and the number of people in each ROI is estimated using a regression function. Finally, an integer programming approach is used to recover the instantaneous count from the set of temporal ROI counts.

\subsection{Crowd segmentation}

Motion segmentation is first applied to the video to focus the counting algorithm on different crowds of interest (e.g., moving in opposite directions). We use a mixture of dynamic textures motion model [14] to extract the regions with different crowd flows. The video is divided into a set of spatiotemporal video cubes, from which a mixture of dynamic textures is learned using the EM algorithm [14]. The motion segmentation is then formed by assigning video patches to the most likely dynamic texture component. Static or very slow moving pedestrians will not be included in the motion segmentation, which is desirable, since the counting algorithm should ignore people who have stopped on the line, in order to avoid double counting.

\subsection{Line sampling and temporal ROI}

In contrast to flow-mosaicking [1], we use line sampling with a fixed line-width to obtain the temporal slice image. As shown in Fig. 4, the input video image and its corresponding segmentation are sampled at the same line per

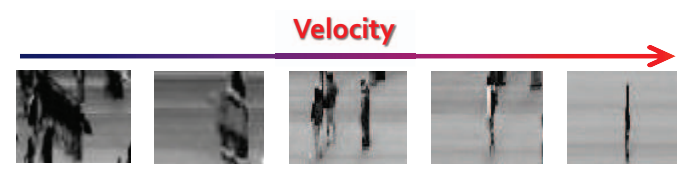

Figure 5. Temporal slices of pedestrians with different velocities.

(a)

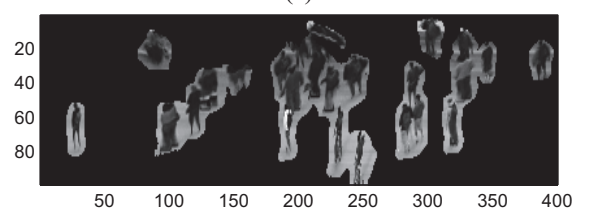

(b)
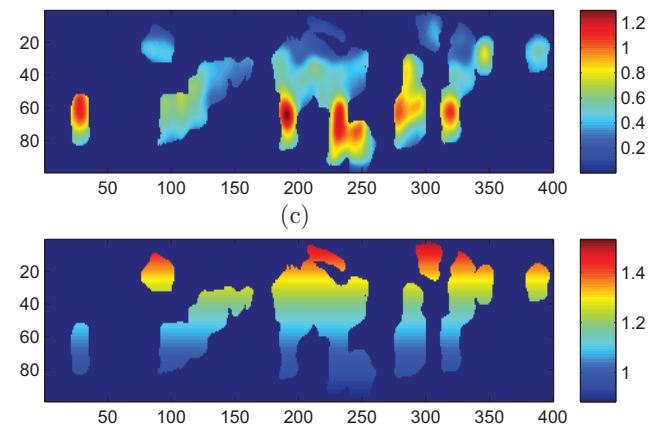

Figure 6. a) temporal-slice image, and its b) temporal and c) spatial weighting maps.

frame. The sampled image slices and segment slices are collected to form the temporal slice image and temporal slice segmentation, where each column in the slice image corresponds to the LOI at a given time. To obtain the temporal ROIs, a sliding window is moved horizontally across the slice image, using a stepsize of one pixel.

\subsection{Feature extraction}

Features are extracted from each crowd segment in each temporal ROI. In this paper, we consider both low-level global features and local features. We use the 30 global features from [3], which measure various properties of the segment, and its internal edges and texture (see Table 1).

For the local features, we use our proposed local HOG features and bag-of-words model. A set of local HOG features is extracted from densely sampled patches over the crowd segment in the temporal ROI. The set of local HOGs is then summarized using the bag-of-words model, as described in Section 3, resulting in a single feature vector for each crowd segment for each ROI.

\subsection{Spatial-temporal normalization}

Because the temporal slice image is generated using a fixed-width line, the width of a person will change with its velocity. In particular, people moving slowly across the LOI will appear wider than those moving fast, as illustrated in Fig. 5. Hence, temporal normalization is required during 
Table 1. Spatial-temporal normalization for low level features

\begin{tabular}{|c|c|c|c|}
\hline Group & Features & Dimension & Weighting strategy \\
\hline & area & 1 & $w_{p} w_{v}$ \\
segment & perimeter & 1 & $\sqrt{w_{p} w_{v}}$ \\
features & perimeter-area ratio & 1 & $\sqrt{w_{p} w_{v}}$ \\
$(10)$ & perimeter edge orientation & 6 & $\sqrt{w_{p}^{2} \cos ^{2} \theta+w_{v}^{2} \sin ^{2} \theta}$ \\
& blob number & 1 & $\mathrm{~N} / \mathrm{A}$ \\
\hline edge & edge length & 1 & $\sqrt{w_{p} w_{v}}$ \\
features & edge orientation & 6 & $\sqrt{w_{p}^{2} \cos ^{2} \theta+w_{v}^{2} \sin ^{2} \theta}$ \\
$(8)$ & edge Minkowski & 1 & $\sqrt{w_{p} w_{v}}$ \\
\hline texture & texture homogeneity & 4 & $\sqrt{w_{p} w_{v}}$ \\
features & texture energy & 4 & $\sqrt{w_{p} w_{v}}$ \\
$(12)$ & texture entropy & 4 & $\sqrt{w_{p} w_{v}}$ \\
\hline
\end{tabular}

feature extraction to adjust for the speed of the person (features). A temporal weight map $w_{v}(x, y)$ is formed from the tangent velocity of each LOI pixel, estimated with optical flow [15] (see Fig. 6b). Faster moving people have higher weights, since their features will be present for less time.

In addition to the temporal normalization, the features must also be normalized to adjust for perspective effects of the angled camera. We follow [2] to generate the spatial perspective weight map $w_{p}(x, y)$ (see Fig. 6c).

Both weighting maps are applied when extracting lowlevel features from the image, yielding a spatio-temporal normalization summarized in Table 1. For the area feature, each pixel is weighted by $w_{p} w_{v}$, and for most edge and texture features, the weighting of $\sqrt{w_{p} w_{v}}$ is applied on each pixel. The edge orientation features are sensitive to a particular edge angle $\theta \in\left\{0^{\circ}, 30^{\circ}, 60^{\circ}, 90^{\circ}, 120^{\circ}, 150^{\circ}\right\}$, and hence a weight of $\sqrt{w_{p}^{2} \cos ^{2} \theta+w_{v}^{2} \sin ^{2} \theta}$ is used to readjust the contributions between $w_{v}$ and $w_{p}$. For example, when the edge is oriented horizontally $\left(\theta=90^{\circ}\right)$, only the temporal weight is applied, since there is no component of the edge in the spatial direction.

For the local HOG feature, we resize the image patch by scaling the height and width by $w_{p}$ and $w_{v}$. This normalizes the local HOG feature extraction to a common reference size. However, normalization of the local HOG features is not necessary; our experimental results show similar performance between local HOG with and without spatiotemporal normalization, which indicates the robustness of the feature to perspective and velocity variations.

Finally, note that flow mosaicking [1] performs temporal normalization by sampling the LOI using a variable linewidth, where the current width is based on the average speed of the crowd blob. Because the same line-width must be applied to the whole blob, blobs containing both fast and slow people will not be normalized correctly. In contrast to [1], we use a fixed line-width and per-pixel temporal normalization, which can better handle large crowd blobs with people moving at different speeds (e.g., in Figs. 6a and $6 b)$.

\subsection{Count Regression}

For each temporal ROI, the count in each crowd segment of the ROI is predicted using a regression function that directly maps between the feature vector (input) and the number of people in the crowd segment (output). Gaussian process regression (GPR) [8] has shown promising results for the people counting task [2]. However, pedestrian counts are discrete non-negative integer values, and hence it is not suitable to use GP regression, which models continuous real-valued outputs. Aiming to take full advantage of Bayesian inference, we use Bayesian Poisson regression [3], which directly learns a regression function with discrete integer outputs. We use the combination of RBF kernel and linear kernel, which yielded the best performance, compared to the single RBF kernel, linear, Bhattacharyya, histogram intersection, and Chi-squared-RBF kernels. Fig. 7a presents an example of the predicted counts for the temporal ROIs, along with the ground-truth.

\subsection{Instantaneous count estimation}

In the final stage, the instantaneous counts on the LOI are recovered from the temporal ROI counts using an integer programming formulation. The $i$ th temporal ROI spans time $i$ through $i+L-1$, where $L$ is the width of the ROI. Let $n_{i}$ be the count in the $i$ th temporal ROI, and $s_{j}$ be the instantaneous count on the LOI at time $j$. The temporal ROI count $n_{i}$ is the sum of the instantaneous counts $s_{j}$, within the temporal window of the ROI,

$$
n_{i}=s_{i}+s_{i+1}+\cdots+s_{i+L-1}=\sum_{k=0}^{L-1} s_{i+k},
$$

Defining the vector of ROI counts $n=\left[n_{1}, \ldots, n_{N}\right]^{T}$ and $s=\left[s_{1}, \ldots, s_{M}\right]^{T}$, where $N$ is the number of temporal ROIs and $M$ is the number of video frames, we have

$$
n=A s,
$$

where $A \in\{0,1\}^{N \times M}$ is an association matrix with entries

$$
a_{i j}= \begin{cases}1, & j \leq i<j+L \\ 0, & \text { otherwise }\end{cases}
$$

Both $n$ and $A$ are known, and hence finding $s$ is a signal reconstruction problem, with non-negative integer constraints on the counts $s_{j}$. We propose to recover the instantaneous counts $s$ using an integer programming problem with a sumsquared reconstruction error

$$
s^{*}=\underset{s}{\operatorname{argmin}}\|A s-n\|^{2} \quad \text { s.t. } s_{j} \in \mathbb{Z}^{+}, \forall j,
$$

where $\mathbb{Z}^{+}$is the set of non-negative integers. We solve (4) using the optimization toolbox [16]. Fig. 7b presents an example of the instantaneous counts recovered from the ROI 
(a)

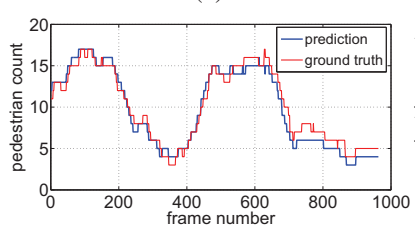

(c)

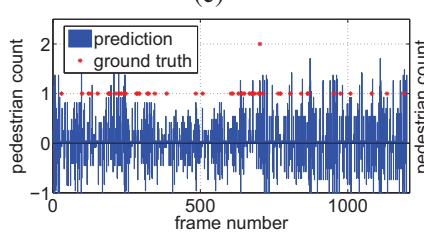

Figure 7. a) temporal ROI counts over time, and the recovered instantaneous count estimates using b) integer programming, c) least-squares, and d) non-negative least-squares.

counts in Fig. 7a with integer programming. The predicted instantaneous counts are close to the ground-truth people crossing the line.

Another possible solution is to relax the non-negative integer constraints on $s_{j}$. Letting $s_{j}$ be a real number, we obtain a standard least-squares formulation, with $s=A^{\dagger} n$, where $A^{\dagger}$ is the pseudo-inverse of $A$. However, the recovered $s_{j}$ will be both positive and negative, which are not interpretable as counts (see Fig. 7c). Enforcing a non-negative real constraint, $s_{j} \geq 0$, yields a non-negative least-squares problem, with the recovered $s_{j}$ more similar to counts (see Fig. 7d). However, this method causes localization errors, where a single count is split into several small real-valued counts in its neighborhood (see arrow in Fig. 7d). Using the integer programming formulation fixes these localization problems (Fig. 7b arrow).

\section{Experiments}

In this section, we present experimental results on using the proposed LOI algorithm on two crowd datasets.

\subsection{Pedestrian datasets}

We consider two datasets in our experiments, the UCSD people counting dataset [2] and the LHI pedestrian dataset [17]. An example frame from the UCSD dataset is shown in Fig. 8a. The video is captured by a stationary digital camcorder with an angled viewpoint over a walkway at UCSD. The dataset contains 2000 video frames, with a frame size of $238 \times 158$ and frame rate of $10 \mathrm{fps}$. The LHI dataset contains three types of video, categorized by the camera tilt angle. In our experiments, we use the 3-3 video with 40 degrees camera tilt angle, which is the most challenging video in LHI due to the large amounts of occlusion. An example frame is displayed in Fig. 8b. The 3-3 video has frame size $352 \times 288$. (a)

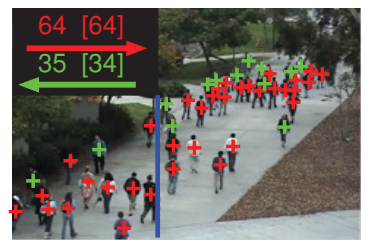

(b)

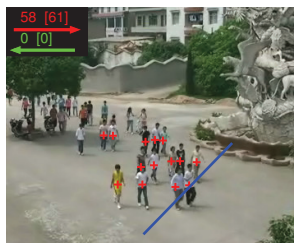

Figure 8. Examples of input video with line position for a) UCSD dataset, and b) video 3-3 of LHI dataset.

\subsection{Experimental setup}

For the UCSD dataset, we follow the experimental protocol in [2], where the training set consists of 800 frames (frames 600 to 1399), and the remaining 1200 frames are used as the test set for validation. For the LHI dataset, the training set is the first 800 frames and the following 1200 frames are the test set. The LOI positions are also shown in Fig. 8. For UCSD, the crowd was separated into two components moving in opposite directions on the walkway (right and left), using the method described in Section 4.1. For LHI, the crowd is only moving in the right direction.

We estimate the instantaneous and cumulative counts on the LOI using our proposed framework. The length of the sliding window is 238 pixels. We tested both the global lowlevel features [2, 4] and the proposed local HOG features, with and without using spatio-temporal normalization. The regression model is learned from the training set (UCSD or LHI), and predictions made on the corresponding test set. For comparison, we also predict the cumulative counts using the flow-mosaicking algorithm [1]. Both methods are run on the same motion segmentation and optical flow images.

The cumulative counting results are evaluated with the mean squared error (MSE) and the absolute error between the predicted counts and the ground truth number of people, averaged over all frames in the test set. For flow mosaicking, which is blob-based and inherently cannot produce smooth cumulative counts, we also consider a "blob groundtruth" that updates only when the predicted count changes, i.e., when a blob is counted.

A recall-distance curve is used to measure the performance of the instantaneous count predictions. The groundtruth instantaneous count and the predictions are matched pairwise using the Hungarian algorithm to find pairs with minimal temporal distances (i.e., match the red stars with blue lines in Fig. 11b). A recall-distance curve is formed by sweeping a threshold distance $d$, and recording the fraction of pairwise matches with distance less than $d$. The curve represents the accuracy of detecting a person crossing the line within duration $d$ of the ground-truth crossing.

\subsection{Experimental results}

The cumulative counting results are summarized in Table 2, with plots of the cumulative and instantaneous 


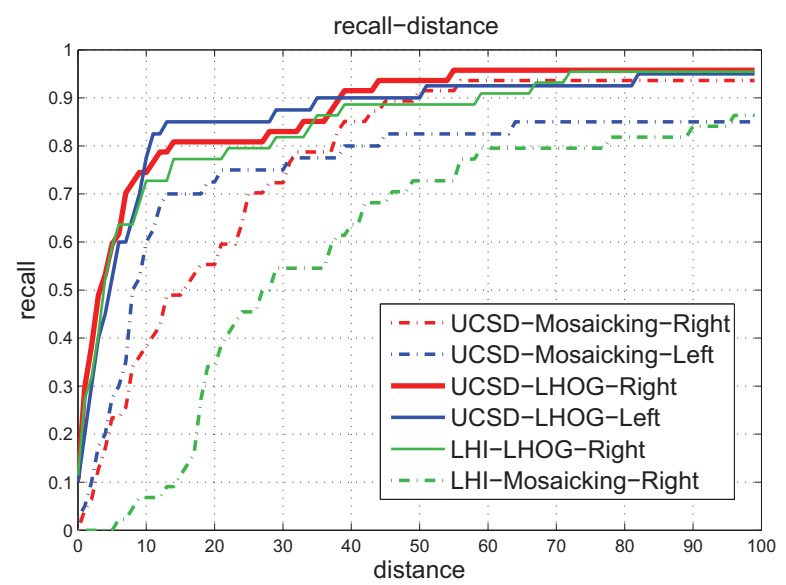

Figure 9. Recall-distance curves on UCSD and LHI datasets.

counts shown in Figs. 11-16. First comparing the different feature sets on the UCSD dataset, the local HOG feature achieves comparable results with the global low-level features $(0.6040$ error vs 0.5342$)$ for the left direction. On the right direction, local $\mathrm{HOG}$ has significantly less error than the global features $(0.6883$ vs 1.5067$)$. Since the right directions contains larger crowds, this suggests that the local HOG features are better at counting the partially-occluded people. Furthermore, the counting error for the local HOG feature is nearly the same when no spatio-temporal normalization is used, increasing slightly from $0.6050 / 0.6883$ to $0.6083 / 0.7100$. On the other hand, the error for the global features increases significantly, e.g., from 1.5067 to 2.4158 for the right direction. This demonstrates that the local HOG feature is robust to perspective and velocity effects, whereas the global features are sensitive to these effects.

Our LOI counting framework using local HOG has lower error than flow-mosaicking (for both the ground-truth and blob ground-truth). Flow-mosaicking has a particularly large error (8.2400) on the right direction. In crowded scenes with large blobs, the flow mosaicking method tends to have high error, which is also shown in Fig. 14 and Fig. 16. Similar results are obtained on the LHI video, demonstrating that our framework achieves lower cumulative counting error than flow-mosaicking.

The recovered instantaneous counts are presented in Fig. 2, and the accuracy is evaluated using the recalldistance curves in Fig. 9. On the UCSD dataset, our method can correctly identify over $80 \%$ of the pedestrians crossing the line within 2 seconds (20 frames, $10 \mathrm{fps}$ ), and on the LHI dataset, almost $90 \%$ within 2 seconds (50 frames, 25 fps). Our method can generate more accurate instantaneous counts than flow mosaicking, which is a "blob-centric" method. For comparison, flow-mosaicking can identify $55 \%$ and $75 \%$ of the pedestrians within 2 seconds on UCSD, and $72 \%$ on LHI.

Finally, Fig. 10a presents the average absolute count-
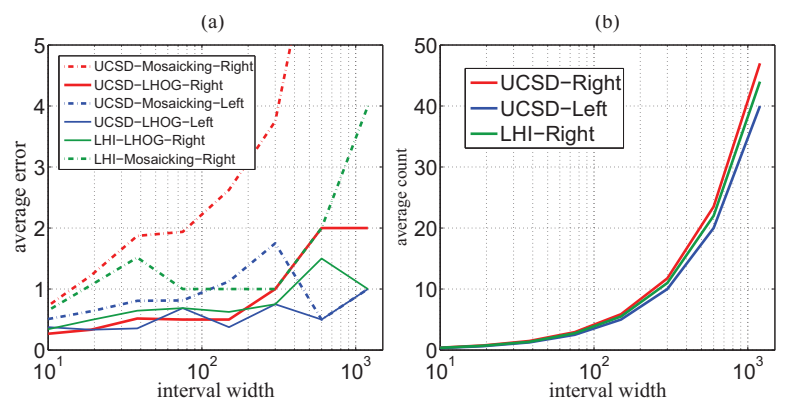

Figure 10. Comparison over different time intervals: a) average error vs interval width; b) average count vs interval width

ing error for varying time intervals (window lengths), and Fig. 10b shows the corresponding average number of ground truth people. For our method, the counting error is relatively stable regardless of the interval width, whereas that of flow-mosaicking increases as the interval width and number of people increases. Two videos of our line counting results on UCSD and LHI datasets can be found in the supplemental material.

\section{Conclusion}

We present a novel line counting framework in this paper, which is based on using integer programming to recover the instantaneous counts on the LOI from ROI counts on a sliding window over the temporal slice image. We validate our framework on two challenging datasets. The results show that, compared with global low-level features, the proposed local HOG feature is more robust to the perspective and object velocity variations, and performs equally well without using spatio-temporal normalization. Moreover, compared with "blob-centric" methods (e.g. flow-mosaicking), our method can generate more accurate instantaneous and cumulative counts, especially in crowded scenes.

\section{Acknowledgments}

The authors thank Yang Cong for the videos from the LHI dataset [17]. This work was supported by the Research Grants Council of the Hong Kong Special Administrative Region, China [CityU 110610].

\section{References}

[1] Y. Cong, H. Gong, S.-C. Zhu, and Y. Tang, "Flow mosaicking: Realtime pedestrian counting without scene-specific learning," in CVPR, 2009, pp. $1093-1100$.

[2] A. B. Chan, Z.-S. J. Liang, and N. Vasconcelos, "Privacy preserving crowd monitoring: Counting people without people models or tracking," in CVPR, 2008, pp. $1-7$.

[3] A. B. Chan and N. Vasconcelos, "Counting people with low-level features and bayesian regression," IEEE Trans. on Image Processing, vol. 21, no. 4, pp. $2160-2177,2012$.

[4] - "Bayesian poisson regression for crowd counting," in ICCV, 2009 , pp. $545-551$. 
Table 2. Cumulative counting results on UCSD and LHI datasets.

\begin{tabular}{|c|c|c|c|c|c|c|c|}
\hline \multirow{2}{*}{ Dataset } & \multirow{2}{*}{$\begin{array}{c}\text { Spatio-temporal } \\
\text { normalization? }\end{array}$} & \multirow{2}{*}{ Method } & \multirow{2}{*}{ Feature } & \multicolumn{2}{|c|}{ Left } & \multicolumn{2}{|c|}{ Right } \\
\hline & & & & Error & MSE & Error & MSE \\
\hline \multirow{10}{*}{ UCSD } & \multirow{6}{*}{ Yes } & \multirow{4}{*}{ Ours } & local HOG & 0.6040 & $\mathbf{0 . 6 2 5 7}$ & 0.6883 & 0.9550 \\
\hline & & & segment & 1.2233 & 2.5833 & 4.5367 & 22.9717 \\
\hline & & & edge & 3.8417 & 22.3800 & 1.7517 & 3.8150 \\
\hline & & & segment + edge + texture & 0.5342 & 0.6875 & 1.5067 & 3.0967 \\
\hline & & [1] & area + edge length & 1.7233 & 4.4783 & 8.2400 & 93.2033 \\
\hline & & [1](blob GT) & area + edge length & 1.3108 & 2.5808 & 8.3767 & 99.6150 \\
\hline & \multirow{4}{*}{ No } & \multirow{2}{*}{ Ours } & local HOG & 0.6083 & 0.6867 & 0.7100 & 1.0817 \\
\hline & & & segment +edge + texture & 0.9958 & 1.4225 & 2.4158 & 7.6342 \\
\hline & & [1] & area + edge length & 1.8583 & 4.9317 & 11.0108 & 167.3308 \\
\hline & & [1](blob GT) & area + edge length & 1.4458 & 2.8575 & 11.1492 & 176.6292 \\
\hline \multirow{3}{*}{ LHI } & \multirow{2}{*}{ Yes } & [1] & area + edge length & - & - & 3.3400 & 17.8533 \\
\hline & & [1](blob GT) & area + edge length & - & - & 2.4058 & 6.5308 \\
\hline & No & Ours & local HOG & - & - & $\mathbf{0 . 8 2 5 0}$ & 1.6350 \\
\hline
\end{tabular}
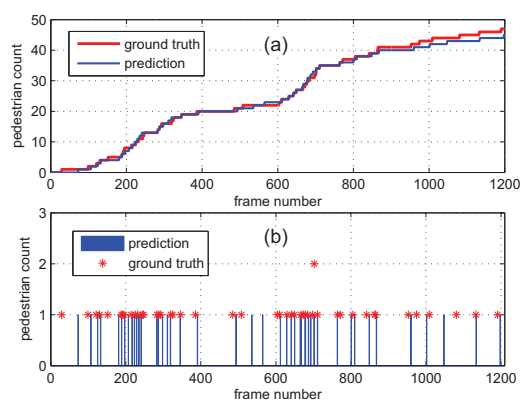

Figure 11. Local HOG - UCSD right
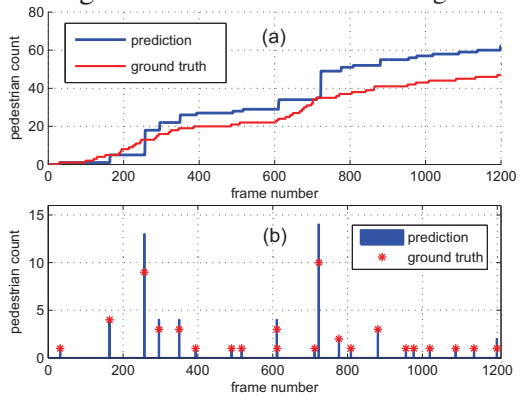

Figure 14. Flow mosaicking - UCSD right
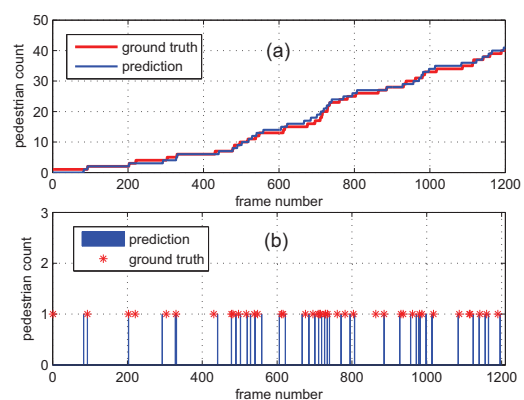

Figure 12. Local HOG - UCSD left
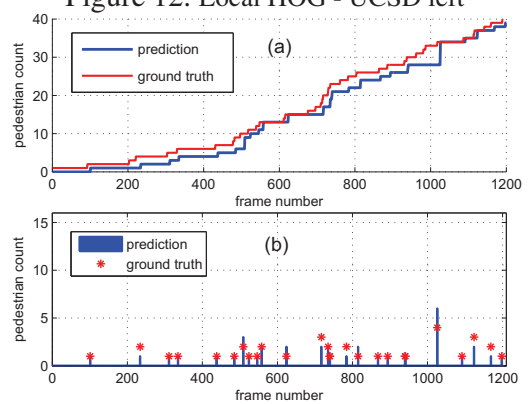

Figure 15. Flow mosaicking - UCSD left
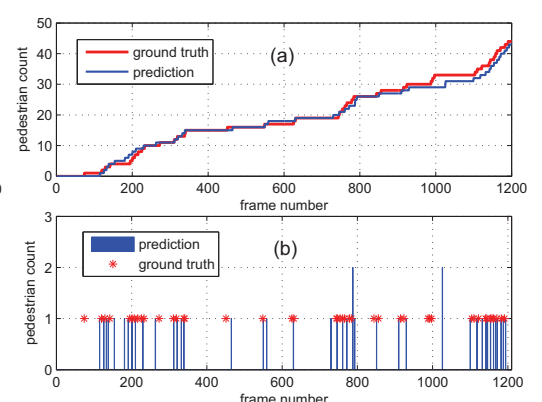

Figure 13. Local HOG - LHI 3-3 right
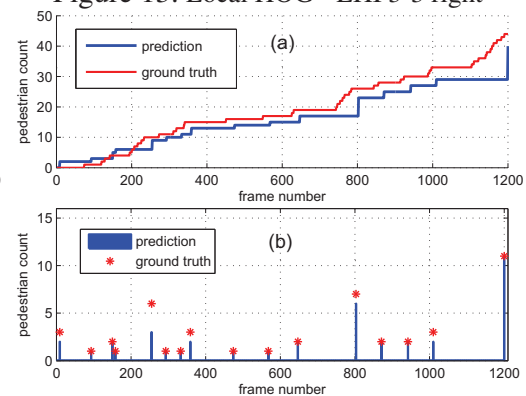

Figure 16. Flow mosaicking - LHI 3-3 right
[5] D. Kong, D. Gray, and H. Tao, "A viewpoint invariant approach for crowd counting," in ICPR, 2006, pp. $1187-90$.

[6] D. Ryan, S. Denman, C. Fookes, and S. Sridharan, "Crowd counting using multiple local features," in Digital Image Computing: Techniques and Applications, 2009, pp. $81-88$.

[7] F. Lv, T. Zhao, and R. Nevatia, "Camera calibration from video of a walking human," IEEE Trans. on PAMI, vol. 28, no. 9, pp. 1513 $-1518,2006$.

[8] C. E. Rasmussen and C. K. I. Williams, Gaussian Processes for Machine Learning. MIT Press, 2006.

[9] V. Lempitsky and A. Zisserman, "Learning to count objects in images," in Advances in NIPS, 2010.

[10] D. G. Lowe, "Distinctive image features from scale-invariant keypoints," IJCV, vol. 60, pp. 91-110, 2004.

[11] N. Dalal and B. Triggs, "Histograms of oriented gradients for human detection," in CVPR, vol. 1, 2005, pp. $886-93$.

[12] B. Wu and R. Nevatia, "Detection of multiple, partially occluded humans in a single image by bayesian combination of edgelet part detectors," in ICCV, vol. 1, 2005, pp. $90-7$.

[13] P. Felzenszwalb, D. McAllester, and D. Ramanan, "A discriminatively trained, multiscale, deformable part model," in CVPR, 2008, pp. 1 $-8$.

[14] A. B. Chan and N. Vasconcelos, "Modeling, clustering, and segmenting video with mixtures of dynamic textures," IEEE Trans. on PAMI, vol. 30, no. 5, pp. $909-926,2008$.

[15] A. Bruhn, J. Weickert, and C. Schnrr, "Lucas/kanade meets horn/schunck: Combining local and global optic flow methods," IJCV, vol. 61, pp. 211-231, 2005.

[16] "Ibm ilog cplex optimizer," http://www-01.ibm.com/software/ integration/optimization/cplex-optimizer/.

[17] B. Yao, X. Yang, and S.-C. Zhu, "Introduction to a large-scale general purpose ground truth database: methodology, annotation tool and benchmarks," in In 6th Int'l Conf on EMMCVPR, 2007, pp. 169-183. 